\title{
Pacific
}

Journal of

Mathematics

\section{FINITELY GENERATED COHOMOLOGY HOPF ALGEBRAS} AND TORSION

\author{
JAMES PEICHENG LiN
}




\title{
FINITELY GENERATED COHOMOLOGY HOPF ALGEBRAS AND TORSION
}

\author{
JAMES P. LIN
}

\begin{abstract}
$H$-spaces $X$ whose mod $p$ cohomology is finitely generated as an algebra are studied. Even generators of infinite height lie in degrees $2 p^{j}$ for $j \geq 0$. If $H^{*}\left(X ; \mathbb{Z}_{p}\right)$ is not finite dimensional, then $H^{*}(X ; \mathbb{Z})$ must have $p$ torsion of all orders if $X$ is two connected.
\end{abstract}

\section{Introduction.}

In this note we begin a study of $H$-spaces whose cohomology $\bmod p$ is not finite dimensional, but is finitely generated as an algebra. We study these $H$-spaces by studying the structure of their Borel decompositions. From the Borel structure theorem, if the mod $p$ cohomology of an $H$-space is nonfinite, either there are an infinite number of algebra generators or there are elements of infinite height. We prove the following:

Theorem A. Let $A$ be a mod p cohomology Hopf algebra admitting an action of the Steenrod algebra. If $A$ is finitely generated as an algebra, then the generators of infinite height lie in degrees of the form $2 p^{j}$, for $j \geq 0$.

In the next theorem we show that nonfinite $H$-spaces must have unbounded $p$-torsion in their cohomology.

Theorem B. Let $X$ be a two-connected $H$-space and suppose $p^{r} H^{*}(X ; \mathbb{Z})$ is torsion-free for some $r>0$. Then if $H^{*}\left(X ; \mathbb{Z}_{p}\right)$ is finitely generated as an algebra, then it is finite dimensional.

Theorem C. Let $X$ be a two-connected $H$-space with $H^{*}\left(X ; \mathbb{Z}_{p}\right)$ finitely generated as an algebra, but not finite dimensional. Then $\beta_{1} Q H^{\text {even }}\left(X ; \mathbb{Z}_{p}\right) \neq$ 0 , where $\beta_{1}$ is the first cohomology Bockstein.

Theorem D. Let $X$ be a two-connected $H$-space with $H^{*}\left(X ; \mathbb{Z}_{p}\right)$ finitelygenerated as an algebra, but not finite dimensional. Then $H^{*}(X ; \mathbb{Z})$ has p-torsion of all orders.

Corollary E. Let $X$ be a homotopy associative $H$-space with $H^{*}\left(X ; \mathbb{Z}_{p}\right)$ finitely generated as an algebra and primitively generated. If $p$ is an odd 
prime, all even generators lie in degrees $2 p^{j}, j \geq 0$, and for every primitive even generator $x$, of degree greater than $2, \beta_{1} x \neq 0$.

One should note that the three-connective cover of a Lie group is an example of an $H$-space whose cohomology $\bmod p$ is finitely generated as an algebra, but not finite dimensional. Other examples are $K\left(\mathbb{Z}_{p} r, 1\right) s$ and $K(\mathbb{Z}, 2) s$. Presently these are the only examples known to the author. From this, one might conjecture that all $H$-spaces whose cohomology mod $p$ is finitely generated, but not finite has the $\bmod p$ cohomology of a product of a finite $H$-space with copies of three-connective covers of Lie groups and $K\left(\mathbb{Z}_{p} r, 1\right) s$ and $K(\mathbb{Z}, 2) s$.

There are a number of results related to Theorems A, B, C, D. Throughout this discussion, let $X$ be an $H$-space whose $\bmod p$ cohomology is finitely generated as an algebra. If the Bockstein $\beta_{1}$ vanishes, Lin [L1] shows if $p$ is odd, all even generators lie in degree 2 . If $X$ is homotopy commutative and homotopy associative, Slack $[\mathbf{S}]$ shows $X$ is $\bmod 2$ equivalent to a generalized Eilenberg MacLane complex with homotopy groups in degrees 1 and 2 . If $X$ is the loops on an $a_{p}$ space, Lin [L2] proves the same result for $p$ odd.

Throughout the entire paper, all spaces will have the homotopy type of a connected $\mathrm{CW}$ complex with finitely many cells in each degree. Unless otherwise specified, all cohomology or homology modules will be understood to be with $\mathbb{Z}_{p}$ coefficients where $p$ is a prime. All Hopf algebras are assumed to be connected and biassociative. We will assume the reader has some familiarity with the concepts of cohomology Hopf algebras. These objects are discussed in detail in [MS].

Finally the author would like to express his appreciation to Mike Slack and Clarence Wilkerson for several useful conversations. In particular, Theorem 2.1 is due to Wilkerson. Also the referee considerably shortened the proof of Theorem A.

\section{$\S 1$. Elements of Infinite Height.}

In this chapter we prove Theorem A using the $T$-functor technology originally due to Lannes $[\mathbf{L}]$. Throughout this chapter, $A$ will be a finitely generated $\bmod p$ cohomology Hopf algebra over the Steenrod algebra.

Proposition 1.1. There exists an integer $N$ such that the $p^{N}$ powers of elements of $A$ form a Hopf subalgebra that has only elements of infinite height.

Proof. Let $\xi: A \rightarrow A$ be the $p$ th power map. Then $\xi^{N} A$ is a finitely generated Hopf algebra over the Steenrod algebra. If the generators of finite height 
have height less than $p^{N}$, then $\xi^{N} A$ is polynomial by the Borel structure theorem.

Following the notation of Lannes, let $V=\left(\mathbb{Z}_{p}\right)^{\ell}$ and $B V=K\left(\left(\mathbb{Z}_{p}\right)^{\ell}, 1\right)$. Recall

$$
H^{*}\left(B V ; \mathbb{Z}_{p}\right) \cong \wedge\left(x_{1}, \ldots, x_{\ell}\right) \otimes \mathbb{Z}_{p}\left[y_{1}, \ldots, y_{\ell}\right]
$$

for $p$ odd where the degrees of the $x_{i} \mathrm{~s}$ is one and the degrees of the $y_{\jmath} \mathrm{s}$ is two. For $p=2$,

$$
H^{*}\left(B V ; \mathbb{Z}_{2}\right)=\mathbb{Z}_{2}\left[x_{1}, \ldots, x_{\ell}\right] \quad \operatorname{deg} x_{\imath}=1 .
$$

Proposition 1.2. Suppose $\xi^{N} A$ embeds as Hopf algebras into $H^{*}\left(B V, \mathbb{Z}_{p}\right)$ for some $\ell>0$. Then the generators of infinite height of $A$ lie in degrees $2 p^{j}$ for $j \geq 0$.

Proof. If the generators of $\xi^{N} A$ lie in degrees $2 p^{k}$ for $k>0$, then the generators of infinite height of $A$ lie in degrees $2 p^{k-N}$ so we are done.

Let $g: \xi^{N} A \rightarrow H^{*}\left(B V ; \mathbb{Z}_{p}\right)$ be a Hopf algebra embedding. Then suppose by induction that all generators of degree less than $m$ in $\xi^{N} A$ lie in degrees twice a power of $p$. Let $B$ be the subalgebra generated by elements of degree less than $m$. Then $B$ is a Hopf algebra and $\xi^{N} A \cong B \otimes \xi^{N} A / / B$ as a left $B$ module and $H^{*}\left(B V ; \mathbb{Z}_{p}\right) \cong g(B) \otimes H^{*}\left(B V ; \mathbb{Z}_{p}\right) / / g(B)$ as a left $g(B)$ module, from [MiMo].

It follows that there is an induced monomorphism

$$
\xi^{N} A / / B \stackrel{\tilde{g}}{\longrightarrow} H^{*}\left(B V ; \mathbb{Z}_{p}\right) / / g(B)
$$

of Hopf algebras. If $x$ is an algebra generator in degree $m$, then $0 \neq \tilde{g}\{x\} \in$ $P\left(H^{*}\left(B V ; \mathbb{Z}_{p}\right) / / g(B)\right)$. It follows that degree $\{x\}=2 p^{k}$ for some $k>0$. Hence $\operatorname{deg} x=2 p^{k}$.

Proposition 1.3. Let $C$ be a polynomial algebra over $\mathcal{A}(p)$ with finitely many generators of even degrees. There is an embedding of Hopf algebras over the Steenrod algebra $C \rightarrow H^{*}\left(B V ; \mathbb{Z}_{p}\right)$ for any appropriately chosen $V$.

Proof. By the Adams Wilkerson embedding theorem $[\mathbf{A W}]$, there is an embedding of domains $f: C \rightarrow H^{*}\left(B T^{n} ; \mathbb{Z}_{p}\right)$. Follow this by the map $H^{*}\left(B T^{n} ; \mathbb{Z}_{p}\right) \rightarrow H^{*}\left(B \mathbb{Z}_{p}^{n} ; \mathbb{Z}_{p}\right)$ and call the composite $g: C \rightarrow H^{*}\left(B \mathbb{Z}_{p}^{n} ; \mathbb{Z}_{p}\right)$. By [DW, Thm. 4.1, (1) $\Rightarrow(2)], T_{g}^{V}(C) \cong T_{\phi}^{V}(C)$. By [DW, Lemma 4.5] $T_{\phi}^{V}(C) \cong C$. Finally by [DMW, Props. 3.5, 3.6], $T_{g}^{V}(C) \rightarrow H^{*}\left(B V ; \mathbb{Z}_{p}\right)$ is monic, and hits the smallest $\mathcal{A}(p)$ Hopf algebra containing $g(C)$.

Proof of Theorem A. Theorem A now follows from Propositions 1.2 and 1.3.

\section{Corollary 1.4.}

(1) $\xi^{N} A$ is primitively generated. 
(2) There is a map of Hopf algebras $A \rightarrow H^{*}\left(B V ; \mathbb{Z}_{p}\right)$ which is monic when restricted to the $p^{N}$ powers of $A$.

Proof. $\xi^{N} A$ may be considered a subHopf algebra of $H^{*}\left(B V ; \mathbb{Z}_{p}\right)$, which is primitively generated. Hence, so is $\xi^{N} A$. This proves (1). (2) follows since $H^{*}\left(B V ; \mathbb{Z}_{p}\right)$ is an unstable injective in the category of unstable algebras.

\section{$\S 2$. Torsion and Finite Generation.}

In this chapter we develop the connection between $p$-torsion in $H^{*}(X ; \mathbb{Z})$ and finite generation of the Hopf algebra $H^{*}\left(X ; \mathbb{Z}_{p}\right)$, for $X$ an $H$-space. The following result is due to Wilkerson.

Theorem 2.1. Let $A$ be a differential cohomology algebra mod $p$ and suppose $A$ is finitely generated as an algebra. Then the homology of $A$ is also finitely generated as an algebra.

Proof. Consider $A$ as a left module over the subring $\xi A$ of $p$ th powers. Then the differential $d$ is a $\xi A$-module map and $A$ is finitely generated as a $\xi A$ module. $\xi A$ is Noetherian, so $A$ is a Noetherian $\xi A$-module. Hence the cycles $Z(A)$ and boundaries $B(A)$ are also finitely generated $\xi A$ modules. Hence the homology $H(A)$ is a finitely generated $\xi A$-module and algebra generators of $H(A)$ may be chosen from $Q(\xi A)$ and the module generators of $Z(A)$.

Theorem 2.2. Let $X$ be an $H$-space with $H^{*}\left(X ; \mathbb{Z}_{p}\right)$ finitely generated as an algebra. If $p^{r} H^{*}(X ; \mathbb{Z})$ is torsion free, than $\beta_{s} Q E_{s}^{\text {even }}$ is decomposable for $s \geq 1$ where $E_{s}$ is the sth term of the Bockstein spectral sequence.

Proof. By a theorem of Browder [B] (infinite implications) given $\bar{x} \in Q E_{s}^{2 n}$ with $\beta_{x} \bar{x}=\bar{y} \neq 0$ indecomposable, either there is a new generator $\bar{x}_{1} \in$ $Q E_{s}^{2 n p}$ with $\beta_{s} \bar{x}_{1}=\bar{y}_{1} \neq 0$ or $\beta_{s+1}\left\{x^{p}\right\}=\left\{x^{p-1} y\right\} \neq 0$ in $Q E_{s+1}$. Now if $p^{r} H^{*}(X ; \mathbb{Z})$ is torsion free, $E_{r}=E_{\infty}$. Let $r$ be the smallest integer such that $E_{r}=E_{\infty}$. Then for some $s<r$ there must be an infinite sequence of nontrivial even generators $\bar{x}, \bar{x}_{1}, \bar{x}_{2}, \cdots$ in $Q E_{s}^{\text {even }}$. But $H^{*}\left(X ; \mathbb{Z}_{p}\right)$ is finitely generated as an algebra. Hence by Theorem 2.1 each $E_{s}$ is also finitely generated as an algebra. It follows that we must have $\beta_{s} Q E_{s}^{\text {even }}$ is decomposable for $s \geq 1$.

Proof of Theorem B and Theorem $\mathrm{C}$. Since $H^{*}\left(X ; \mathbb{Z}_{p}\right)$ is finitely generated as an algebra, the algebra generators of infinite height lie in degrees $2 p^{j}$ for $j \geq 0$ by Theorem A. Further $j \geq 1$ since $X$ is two-connected. Therefore 
it suffices to show there are no generators of infinite height. For $p$ odd, by Theorem 1.3.3 of [L3], and the fact that $\beta_{1} H^{\text {even }}\left(X ; \mathbb{Z}_{p}\right)$ is decomposable (by Theorem 2.2), it follows

$$
Q H^{2 p^{j}}\left(X ; \mathbb{Z}_{p}\right)=\mathcal{P}^{1} Q H^{2 p^{j}-2 p+2}\left(X ; \mathbb{Z}_{p}\right) .
$$

If $H^{*}\left(X ; \mathbb{Z}_{p}\right)$ has a generator of infinite height, let $x$ be such an element of lowest degree. Then by (2.1) and Theorem A,

$$
x=\mathcal{P}^{1} y+d \quad \text { where } d \text { is decomposable. }
$$

By choice of $x, y$ and $d$ both have finite height. Hence $x$ has finite height. This is a contradiction, so $H^{*}\left(X ; \mathbb{Z}_{p}\right)$ has no generators of infinite height. The Borel structure theorem implies $H^{*}\left(X ; \mathbb{Z}_{p}\right)$ is finite dimensional.

For $p=2$, if $H^{*}\left(X ; \mathbb{Z}_{2}\right)$ is not finite dimensional, let $x$ be a Borel generator of infinite height of highest degree, say $2^{j}$. The Adem relations imply for $j>1$,

$$
S q^{2^{3}+1}=S q^{2^{j}} S q^{1}+S q^{2} S q^{1} S q^{2^{j}-2}
$$

We have $j>1$ since $X$ is two-connected. Then since $S q^{1}=\beta_{1}$ we have by Theorem 2.2,

$$
S q^{1} H^{2^{j}}\left(X ; Z_{2}\right) \text { and } S q^{1} S q^{2^{j}-2} H^{2^{j}}\left(X ; \mathbb{Z}_{2}\right) \text { are decomposable. }
$$

We now apply Theorem 1.3 of [L3]. Let $B(m)$ be the $A(2)$ subHopf algebra generated by elements of degree less than or equal to $m$. By induction on $m$, assume $B(m)$ is finite dimensional, and $\bar{\triangle} x \in B(m) \otimes B(m), x \notin B(m)$. By Theorem 1.3 of $[\mathbf{L 3}]$ there is an element $\phi(x) \in H^{2^{j+1}}\left(X ; \mathbb{Z}_{2}\right)$ with

$$
\bar{\triangle} \phi(x)=x \otimes x+\operatorname{im} S q^{2^{j}}+\operatorname{im} S q^{2}+z
$$

where $z \in H^{*} \otimes I(B(m)) H^{*}+H^{*} I(B(m)) \otimes H^{*}$.

We will show $\phi(x)$ is a generator of infinite height. This will contradict the fact that $x$ is a generator of highest degree of infinite height.

We first show $\phi(x)$ is indecomposable. Consider the subalgebra $B$ generated by $\xi H^{*}+\operatorname{im} S q^{1}+\operatorname{im} S q^{2}+B(m)$. Then $x$ has non-zero projection in $Q\left(H^{*}(X) / / B\right)$ since $\bar{x}$ is indecomposable and elements of im $S q^{1}+\operatorname{im} S q^{2}+$ $B(m)$ have finite height. Therefore, there is a primitive $t \in H_{*}\left(X ; \mathbb{Z}_{p}\right)$ with

$$
\langle t, x\rangle=1, \quad\langle t, B\rangle=0 .
$$

By (2.2), $\left\langle t^{2}, \phi(x)\right\rangle=\langle t, x\rangle^{2}=1$ so $\phi(x)$ is dual to a primitive. Therefore, $\phi(x)$ is indecomposable. 
Now $z$ and $S q^{2}\left(H^{*} \otimes H^{*}\right)$ in degree $2^{j+1}$ both have finite height in $H^{*} \otimes H^{*}$, since $B(m)$ has finite height and $H^{\text {odd }}$ and $S q^{2} H^{4 s+2}$ have finite height for $s \geq 1$, by Theorem A. By (2.2), if $\ell$ is large,

$$
\bar{\triangle} \phi(x)^{2^{\ell}}=x^{2^{\ell}} \otimes x^{2^{\ell}}+\xi^{\ell}\left(\operatorname{im} S q^{2^{j}}\right) .
$$

We claim $\phi(x)^{2^{\ell}} \neq 0$ because $\bar{\triangle} \phi(x)^{2^{\ell}} \neq 0$. If $\bar{\triangle} \phi(x)^{2^{\ell}}=0$ then $x^{2^{\ell}}=$ $y^{2^{\ell+1}}$ for some $y$ so $\left(x-y^{2}\right)$ has height $2^{\ell}$. But a Borel generator has the property that the height of $x$ is less than or equal to the height of $x+d$ where $d$ is decomposable. This is a contradiction, so $\bar{\Delta} \phi(x)^{2^{\ell}} \neq 0$ for all $\ell$ and $\phi(x)$ is a generator of degree higher than $x$ of infinite height. Further, $\phi(x)$ cannot be changed by decomposables to have finite height. So there must be a Borel generator of degree greater than degree of $x$ of infinite height. This is a contradiction. We conclude $H^{*}\left(X ; \mathbb{Z}_{2}\right)$ is finite dimensional.

To prove Theorem $\mathrm{C}$, notice the above argument shows if $\beta_{1} Q H^{\text {even }}\left(X ; \mathbb{Z}_{p}\right)$ $=0$ then if $H^{*}\left(X ; \mathbb{Z}_{p}\right)$ is finitely generated as an algebra, there are no elements of infinite height.

Proof of Theorem D. By Theorem $\mathrm{C}$ there exists an even degree generator $x \in H^{2 n}\left(X ; \mathbb{Z}_{p}\right)$ with $\beta_{1} x=y$ where $y$ is indecomposable. By infinite implications we have either $E_{r}$ is not finitely generated as an algebra or there is an even generator $z$ in $E_{r+1}$ with $\beta_{r+1} z$ indecomposable. By Theorem 2.1 we must have $\beta_{r+1} \neq 0$ for every $r$. This implies there exists $p$ torsion of all orders.

Proof of Corollary E. By $\left[\mathrm{Z} 1\right.$, Thm. C] $H^{*}\left(X ; \mathbb{Z}_{p}\right)$ is free commutative, so by Theorem $\mathrm{A}$, all even generators lie in degrees $2 p^{j}$. Now suppose there is an even primitive generator $x \in P H^{2 p^{j}}\left(X ; \mathbb{Z}_{p}\right)$ with $\beta_{1} x=0$, and $j>0$. Then the Adem relations imply

$$
\beta_{1} \mathcal{P}^{p^{3}}=c_{1} \mathcal{P}^{p^{j}} \beta_{1}+c_{2} \mathcal{P}^{1} \beta_{1} \mathcal{P}^{p^{j}-1}
$$

where $c_{1}, c_{2} \in \mathbb{Z}_{p}$.

We have $\mathcal{P}^{p^{3}-1} x$ is decomposable primitive, hence it is zero since all primitives lie in degrees $2 p^{\ell}, \ell \geq 0$. Therefore by [Z2], there is a secondary operation $\phi(x)$ with

$$
\bar{\triangle} \phi(x)=x \otimes \cdots \otimes x+\operatorname{im} \mathcal{P}^{p^{j}}+\operatorname{im} \mathcal{P}^{1} \beta .
$$

Since $x \notin \operatorname{im} \mathcal{P}^{1}$, there exists a $t \in P H_{*}\left(X ; \mathbb{Z}_{p}\right)$ with

$$
\langle t, x\rangle \neq 0 \quad \text { and } \quad\left\langle t, \operatorname{im} \mathcal{P}^{1}\right\rangle=0 .
$$


It follows that

$$
\left\langle t^{p}, \phi(x)\right\rangle \neq 0, \quad \text { so } \quad t^{p} \neq 0 .
$$

But $H_{*}\left(X ; \mathbb{Z}_{p}\right)$ has no $p$ th powers since $H^{*}\left(X ; \mathbb{Z}_{p}\right)$ is primitively generated. We conclude $\beta_{1} x \neq 0$ for every primitive even generator of degree greater than 2 .

\section{References}

[AW] J.F. Adams and C. Wilkerson, Finite $H$-spaces and algebras over the Steenrod algebra, Ann. of Math., 111 (1980), 95-143.

[B] W. Browder, Torsion in H-spaces, Ann. of Math., 74 (1961), 24-51.

[DMW] W. Dwyer, H. Miller and C. Wilkerson, Homotopical uniqueness of classifying spaces, Topology, 31 (1992), 29-45.

[DW] W. Dwyer and C. Wilkerson, Spaces of null homotopic maps, Asterisque, 191 (1990), 97-108.

[H] J. Hubbuck, Finitely generated cohomology Hopf algebras, Topology, 9 (1970), 205210.

[L] J. Lannes, Sur la cohomologie modulo $p$ des p-groupes Abeliens elementaires, in Homotopy Theory, Proc. Durham Symp., 1985, eds. Rees and Jones, Cambridge Univ. Press, Cambridge 1987.

[L1] J. Lin, H-spaces without simple torsion, J. Pure Applied Algebra, 11 (1977), 61-66.

[L2] , Loops of $H$-spaces with finitely generated cohomology rings, submitted to Topology and Its Applications.

[L3] - Torsion in H-spaces, I, Ann. of Math., 103 (1976), 457-487.

[MiMo] J. Milnor and J.C. Moore, On the structure of Hopf algebras, Ann. Math., 81 (1965), 211-264.

[MS] J.C. Moore and L. Smith, Hopf algebras and multiplicative fibrations, I, II, Amer. J. Math., 90 (1968), 752-780, 1113-1150.

[S] M. Slack, A classification theorem for homotopy commutative H-spaces with finitely generated mod 2 cohomology rings, Memoirs AMS, 92 (1991).

[Z1] A. Zabrodsky, Implications in the cohomology of H-spaces, Ill. J. of Math., 16 (1971), 363-375.

[Z2] - Secondary operations in the cohomology of H-spaces, Ill. J. of Math., 15 (1971), 648-655.

Received June 30, 1993 and revised July 28, 1994.

University of California, San Diego

LA JOLLA, CA 92093-0112 




\section{PACIFIC JOURNAL OF MATHEMATICS}

Volume $172 \quad$ No. $1 \quad$ January 1996

A class of incomplete non-positively curved manifolds

BRIAN BOWDITCH

The quasi-linearity problem for $C^{*}$-algebras

41

L. J. BUNCE and JOHN DAVID MAITLAND WRIGHT

Distortion of boundary sets under inner functions. II

Jose Luis Fernandez Perez, Domingo Pestana and José Rodríguez

Irreducible non-dense $A_{1}^{(1)}$-modules

VJACHESLAV M. FUTORNY

$M$-hyperbolic real subsets of complex spaces

101

Giuliana Gigante, Giuseppe Tomassini and Sergio Venturini

Values of Bernoulli polynomials

ANDREW GRANVILLE and ZHI-WEI SUN

The uniqueness of compact cores for 3-manifolds

LUKE HARRIS and PETER SCOTT

Estimation of the number of periodic orbits

BOJU JIANG

Factorization of $p$-completely bounded multilinear maps

Christian Le MERdy

Finitely generated cohomology Hopf algebras and torsion

JAMEs Peicheng LiN

The positive-dimensional fibres of the Prym map

JUAN-CARLOS NARANJO

Entropy of a skew product with a $Z^{2}$-action

KYEWON KOH PARK

Commuting co-commuting squares and finite-dimensional Kac algebras

TAKASHI SANO

Second order ordinary differential equations with fully nonlinear two-point boundary 255 conditions. I

H. BEVAN THOMPSON

Second order ordinary differential equations with fully nonlinear two-point boundary conditions. II

H. BEVAN THOMPSON

The flat part of non-flat orbifolds

FENG XU 